\title{
Isolated Pediatric Supraglottic Stenosis Managed Using Plasma Ablation: A Case Report
}

\author{
Kanika Arora ${ }^{1}$, Shivjee Prasanth ${ }^{2}$, Sachin Singh ${ }^{3}$, Joseph L Mathew ${ }^{4}$, Ramandeep Virk ${ }^{5}$
}

\begin{abstract}
We describe the technique of plasma ablation (PA) used for releasing supraglottic stenosis (SS). Supraglottic stenosis is associated with the history of airway manipulation or surgery causing chronic laryngeal edema and thereby stenosis. This usually manifests as decannulation failures and often associated with dysphagia and aspiration. A child presented to the emergency with distress following ingestion of green chillis and was intubated. Further developed aspiration pneumonia and fiberoptic endoscopy revealed cricopharyngeal stricture (CPS) and SS. Child was taken up for the release with PA and decannulated successfully. Supraglottic stenosis can be safely treated using PA as an alternative to LASER. Keywords: Decannulation, Dysphagia, Plasma ablation, Supraglottic stenosis.

Journal of Postgraduate Medicine, Education and Research (2020): 10.5005/jp-journals-10028-1369
\end{abstract}

\section{INTRODUCTION}

Supraglottic stenosis (SS) may be congenital or acquired. Congenital stenosis is due to laryngomalacia or webs while acquired is associated with trauma, surgery, radiotherapy, or autoimmune disorders. It is also common in children with associated history of airway manipulation or surgery causing chronic laryngeal edema. ${ }^{1}$

Despite good results of endoscopic procedures, dense cicatricial scar and its tendency to recur require multiple procedures and thereby repeated decannulation failures. Multiple modalities have been tried for the release of such stenosis. One such modality has been discussed in the case report which can be used at centers not having the facility of LASER.

\section{Case Description}

A 10-month-old male child presented to emergency with noisy breathing, sudden onset of paroxysmal cough, and vomiting following ingestion of green chillis. He was intubated and put on ventilator (inflamed and edematous cords); tracheotomy was done on the fifth day due to failed extubation followed by decannulation within 1 week.

Developed stridor after 1 month and was readmitted for aspiration pneumonia. Upper gastrointestinal endoscopy (UGIE) was done for suspected tracheo-esophageal fistula (TEF) but it showed severe cricopharyngeal and glottic stenosis and thereby tracheotomy was redone. Magnetic resonance imaging and bronchocopy showed SS with patent airway.

Patient underwent dilatation of cricopharyngeal stenosis and was started and maintained on oral feeds.

After 9 months, patient underwent repeated bronchoscopy which showed extensive fibrotic tissue obscuring the entire view of larynx (Fig. 1A), but glottis and subglottis were normal as seen through a small opening of $3 \mathrm{~mm}$.

Patient was planned for plasma ablation (PA)-assisted release of supraglottic adhesions. We used the four-hand technique. A $0^{\circ}$ $4 \mathrm{~mm}$ Hopkins Rod nasal endoscope (Karl Storz, Germany), Millers laryngoscope blade, and PA (Smith and Nephew, USA) were used for this procedure.

\footnotetext{
1,2,5 Department of ENT, Postgraduate Institute of Medical Education and Research, Chandigarh, India

${ }^{3,4}$ Department of Pediatrics, Postgraduate Institute of Medical Education and Research, Chandigarh, India
}

Corresponding Author: Ramandeep Virk, Department of ENT, Postgraduate Institute of Medical Education and Research, Chandigarh, India, Phone:+91 9914555666, e-mail: virkdoc@hotmail.com

How to cite this article: Arora K, Prasanth S, Singh S, et al. Isolated Pediatric Supraglottic Stenosis Managed Using Plasma Ablation: A Case Report. J Postgrad Med Edu Res 2020;54(2):62-63.

Source of support: Nil

Conflict of interest: None

Intraoperatively, the right side of epiglottis and right arytenoid were found fused with lateral and posterior pharyngeal wall which was released using PA (Fig. 1B), thereby bringing the normal glottis into view (Fig. 1C). Airway was normal till carina (Fig. 1D). The patient was discharged after successful decannulation with significant improvement in aspiration.

\section{Discussion}

The probable cause of stenosis in this case was either laryngeal edema caused due to ingestion of chillis or chronic aspirations associated with acid reflux or airway manipulation during intubation. There are reports of SS due to alkali or acid ingestion which are usually accompanied by strictures in the gastrointestinal tract. $^{2,3}$

It is accompanied with some degree of dysphagia, strictures, decreased pharyngeal contractions, and poor epiglottic retroversion. ${ }^{4}$

Treatment with LASER, either $\mathrm{CO}_{2}$ or pulsed $\mathrm{KTP}_{1}^{4}$ has been more successful as compared to cold instruments, dilatation, or mitomycin C application. Plasma ablation is a versatile and cheaper alternative to LASER and has been described in this case report. It gives the surgeon ablation, bipolar cautery, suction, and irrigation

() The Author(s). 2020 Open Access This article is distributed under the terms of the Creative Commons Attribution 4.0 International License (https://creativecommons. org/licenses/by-nc/4.0/), which permits unrestricted use, distribution, and non-commercial reproduction in any medium, provided you give appropriate credit to the original author(s) and the source, provide a link to the Creative Commons license, and indicate if changes were made. The Creative Commons Public Domain Dedication waiver (http://creativecommons.org/publicdomain/zero/1.0/) applies to the data made available in this article, unless otherwise stated. 


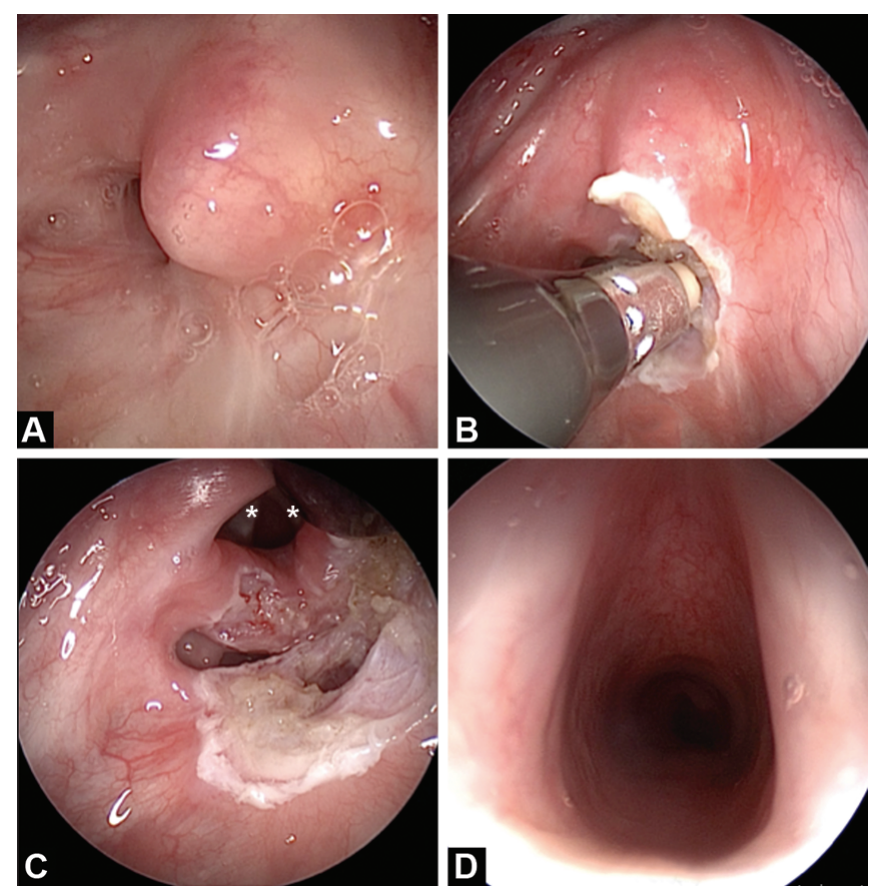

Figs 1A to D: (A) Preoperative endoscopy showing stenosis at the level of supraglottis with small opening into the endolarynx; (B) Use of plasma ablation for releasing the adhesions. The figure shows a microlaryngeal wand; (C) Intraoperative view of coblation release bringing vocal cords ${ }^{*}$ ) into view; (D) Normal glottis and subglottis

in a single sleek device. It creates a plasma field to break molecular bonds and remove tissue. Temperatures range between $40^{\circ} \mathrm{C}$ and $70^{\circ} \mathrm{C}$ with tissue penetration of $1 \mathrm{~mm}$. Flexibility allows the surgeon to reach difficult to visualize areas. It has been successfully used in tracheal stenosis and other airway lesions like vallecular cysts. ${ }^{5-7}$ The use of a four-hand technique makes the surgery simpler, and the endoscope provides a panoramic view. ${ }^{8}$

\section{Conclusion}

Supraglottic stenosis can be safely treated using plasma ablation as an alternative to LASER. Advantages include no risk of airway fire, easy availability, cost effectiveness, and minimal collateral thermal damage. More cases and long-term follow-up are needed for this technique to be effectively compared to other time-tested modalities.

\section{References}

1. Walner DL, Holinger LD. Supraglottic stenosis in infants and children: a preliminary report. Archiv Otolaryngol-Head Neck Surg 1997;123(3):337-341. DOI: 10.1001/archotol.1997.01900030123015.

2. Chen YW, Lai SH, Fang TJ, et al. Pediatric dyspnea caused by supraglottic stenosis: a rare complication of alkali corrosive injury. Europ Archiv Oto-Rhino-Laryngol Head Neck 2006;263(3):210-214. DOI: $10.1007 / \mathrm{s} 00405-005-1011-5$.

3. Berlucchi M, Barbieri D, Garofolo S, et al. Case report: pharyngolaryngeal stenosis in a child due to caustic ingestion treated with transoral CO2 laser microsurgery. Annal Otol, Rhinol Laryngol 2014;123(12):847-851. DOI: 10.1177/0003489414538938.

4. Stevens MS, Chang A, Simpson CB. Supraglottic stenosis: etiology and treatment of a rare condition. Annal Otol, Rhinol Laryngol 2013;122(3):205-209. DOI: 10.1177/000348941312200310.

5. Virk RS, Bansal S, Nayak G. Plasma ablation-assisted endoscopic management of postintubation laryngotracheal stenosis: an alternate tool for management. Otolaryngol-Head Neck Surg 2019;161(6):993-995. DOI: 10.1177/0194599819881439.

6. Virk RS, Mathew JL, Behera S, et al. Endoscopic assisted coblation of congenital vallecular cyst-a novel technique. Indian J Pediatr 2016;83(8):888-889. DOI: 10.1007/s12098-016-2095-1.

7. Gautam V, Basotia A, Virk R. Neonatal vallecular cyst: unusual presentation with near catastrophic respiratory distress and successful treatment using endoscopy-assisted plasma ablation: a case report. J Postgrad Med Edu Res 2020;54(1):15-16. DOI: 10.5005/ jp-journals-10028-1349.

8. Virk RS, Nayak G, Jain D. Miller laryngoscope blade: an aid to pediatric laryngeal surgery. Indian J Otolaryngol Head Neck Surg 2019;71(1):19-21. DOI: 10.1007/s12070-018-1501-6. 